\title{
Enhancing Undergraduate Research Experience with Cutting Edge Technologies
}

\author{
Wenbing Zhao
}

\begin{abstract}
Cleveland State University (CSU) has been striving to create a brand for itself with student engaged learning over the past decade. The engaged-learning effort spans both within classrooms and in extra curriculum activities. Undergraduate research experience is an important component in this effort. In this article, we share our experiences in designing and supervising projects for undergraduate research at CSU as a principal investigator, and the lessons learned in creating projects that are appropriate for undergraduate engineering students. Thanks to the funding from the US National Science Foundation, CSU Provost's Office, CSU Office of Research, and Department of Electrical Engineering and Computer Science, we have been supervising at least one team of CSU undergraduate students in every summer since 2008. We show that undergraduate research projects must be carefully designed at the level of undergraduate students. Inadequate research components in the projects could reduce them to some glorified homework, while over-demanding projects may discourage students' engagement and their interest in science and engineering. The main theme of these projects is the utilization of cutting edge technologies to solve real-world problems, including Microsoft Kinect, Leap Motion Sensor, iOS devices (iPhone/iPad/iPod touch), Android Phone and Tablet, Smart Watches, and other portable programmable sensors. The integration of cutting edge technologies make it possible for us to design projects such that students: (1) will find the projects interesting, (2) can understand the research problems, and (3) can make significant research contributions.
\end{abstract}

Index Terms-Undergraduate research experience, Microsoft Kinect, pebble smart watch, smart phone.

\section{INTRODUCTION}

The undergraduate research experience (URE) has been well recognized as a powerful instructional tool for undergraduate education [1]-[4]. Many studies have shown that URE projects have positive impacts in increasing the graduation rate, attracting more students to consider science and engineering career, and encouraging more students to enter graduate schools.

However, URE is not without controversy. The quality of the research projects matters. If the projects are poorly designed, URE has the risk of becoming "just glorified homework" [5]. Indeed, based on our experiences, designing

Manuscript received April 15, 2016; revised July 2, 2016. This work was supported in part by several NSF REU Supplementary Grants associated with NSF Grant CNS-0821319, and several CSU Undergraduate Summer Research Awards sponsored by the CSU Provost's Office, CSU Office of Research, and CSU Department of Electrical Engineering and Computer Science.

W. Zhao is with the Department of Electrical Engineering and Computer Science, Cleveland State University, Cleveland, OH 44115 USA (e-mail: wenbing@ieee.org). appropriate research projects for undergraduate students is ultimately important for the success of URE. The primary objective of this paper is to share our experiences in designing and supervising projects for undergraduate research in the field of computer science and engineering, and the lessons learned in creating projects that are appropriate for undergraduate engineering students, at the author's institution, Cleveland State University (CSU). We have been supervising at least one team of CSU undergraduate students in every summer since 2008 .

In the following sections, we introduce four projects designed for undergraduate engineering students. A total of eight students (all of them are at junior and senior levels) worked on these projects. All projects were administered in summer and each lasts for 10 weeks. These projects are ordered in chronological order. In the early projects, some of the tasks are too challenging for undergraduate students to complete, or too vague for students to know exactly how to proceed. We recognized these issues in these projects, and started sharpening the design for later projects such that the tasks are very specific and are at a level that is suitable for undergraduate students.

The description of these projects follows the same format. We start by giving an introduction for the significance of the research problem. This is followed by an overview of the research objectives, and specific research duties for the students. We conclude each project by reporting the actual outcomes students managed to accomplish and reflect on the plausible reasons for failed tasks, if any.

This paper makes the following contributions:

- We provide example URE projects in the area of computer science and engineering. In particular, we show how to utilize cutting edge technologies to solve real-world problems. Young students tend to be early adopters of new technologies, such as Microsoft Kinect, Leap Motion Sensor, iOS devices (iPhone/iPad/iPod touch), Android Phone and Tablet, Smart Watches. Based on our observation, they feel genuinely excited about having the opportunity to use them in applied research. The passion would make them more engaged and dedicated to the projects.

- We share the lessons learned in designing URE projects. The nature of research projects dictates that students will have to acquire knowledge beyond their normal curriculum. In fact, an important URE benefit, which is not mentioned in the current literature, is that it encourages students to engage in life-long learning. However, the new knowledge required in the projects should have direct relationship with the curriculum subject matters and there should be a realistic path to 
graph the new knowledge within a short time frame of a few weeks. For example, based on our observation, subjects such as Kalman filters might not be appropriate for URE projects, while students are quite capable of learning a new programming platform such as iOS and Android programming.

\section{PARTICIPATORY SENSING FOR INTELLIGENT TRANSPORTATION SYSTEMS}

This project was designed to engage our undergraduate students in an intelligent transportation systems research involving participatory sensing for data collection. Considering that many drivers nowadays carry smart phones equipped with various sensors such as GPS, accelerometer, camera, and compass, they can provide very valuable data on traffic conditions (such as the speed and trajectory [6]) and the road conditions (such as potholes [7]) and (and hence, participatory sensing). Ultimately, such data can be aggregated, analyzed, and feedback to all drivers for much smoother and safer ride, which is the primary goal of an intelligent transportation system.

\section{A. Research Objectives}

The main goal of the proposed research is to investigate how to use participatory sensing to enrich intelligent transportation systems. While the idea of collecting massive data from vast number of smart phones is rather intuitive and we have seen reports on specific implementations such as pothole detection [7], many open issues must be resolved before participatory sensing can be made useful towards intelligent transportation. The objectives of this research, therefore, are to tackle these open issues. More specifically, the research will focus on the two specific issues:

Issue 1: How to improve the accuracy of the location data by combining readings from various sensors equipped in smart phones. It is well-known that the consumer-grade GPS in smart phones can provide only $5-10 \mathrm{~m}$ accuracy in location readings under the best reception conditions. While such accuracy might be sufficient for many purposes, it is insufficient to pinpoint the specific location of a pothole (so that other drivers could avoid it), and it is even less so to determine the trajectory of vehicles (needed for example to study near-accident incidents around intersections and work zones). By combining the data obtained from accelerometers and the gyroscope, a Kalman filter [8] will be developed to improve the accuracy, hopefully to within the range of several feet.

Issue 2: Develop intuitive, easy-to-use applications that not only capture the participatory sensing data, but also provide useful visual and audio feedbacks (possibly alerts) to drivers on the road and traffic conditions. Furthermore, efforts will also be made to minimize the power consumption when the applications are running.

\section{B. Specific Research Duties of Undergraduate Students}

According to our plan, two undergraduate students will engage in the investigation of using participatory sensing to enrich intelligent transportation systems. More specifically, the students will carry out the following tasks:
1) Develop a participatory sensing application for the iOS platform (for iPhone/iPad/iPod Touch);

2) Develop a participatory sensing application for the Android platform (for most non-apple devices);

3) Develop a Kalman filter to improve the accuracy of the location data.

4) Carry out field tests to assess the effectiveness of the applications and the Kalman filter developed.

\section{Outcomes}

The research objectives in this project are rather aggressive and the completion of the project would require extensive knowledge and skills that are not taught in our undergraduate curriculum. As a result, the two students working in this project could only partially complete the first task (developing an iOS app), the second task (developing an Android app), and the fourth task (field tests). The students were able to find an open source iOS app for GPS data recording (with Google map access) and another iOS app for accelerometer and gyroscope data recording, and integrate them together for an iOS app that can be used to record GPS, accelerometer and gyroscope data together with map information so that the trajectory of the vehicle can be graphically displayed as part of the app. For the Android app although they could also find open source apps for GPS data, accelerometer and gyroscope data recording, they could not obtain access to Google Maps and hence, cannot visually display the driving trajectory. Furthermore, neither the iOS app nor the Android app has the capability of getting realtime input from the driver, for example, when a pothole is spotted. Despite the setbacks, students were able to use both the iOS and Android apps they developed to perform several field trips to collect data driving around CSU.

The Kalman filter appeared to be too hard a mathematical model for students to grasp. They failed to fuse the GPS data and the inertial sensing data using the Kalman filter. However, they did find an alternative method to fuse the data together. Unfortunately, the adjusted trajectory computed using the fused data does not appear to be more accurate, as can be seen from Fig. 1. In fact, the adjusted trajectory looks less smooth than the original trajectory.

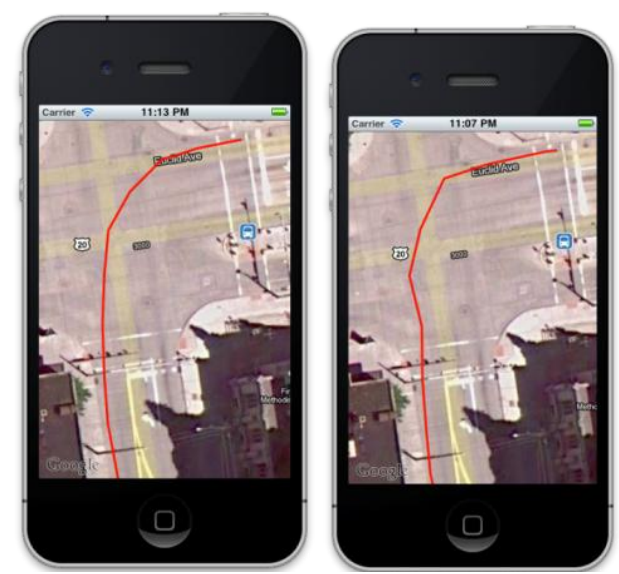

Fig. 1. An example section of the trajectory before adjustment (left) and after adjustment by using the GPS data and the inertial data. The adjusted trajectory is apparently less smooth.

Nevertheless, students did accomplish a number of desirable outcomes that complement their main curriculum: 
- They gained in-depth understanding of the GPS and inertial sensing (accelerometer and gyroscope) technologies.

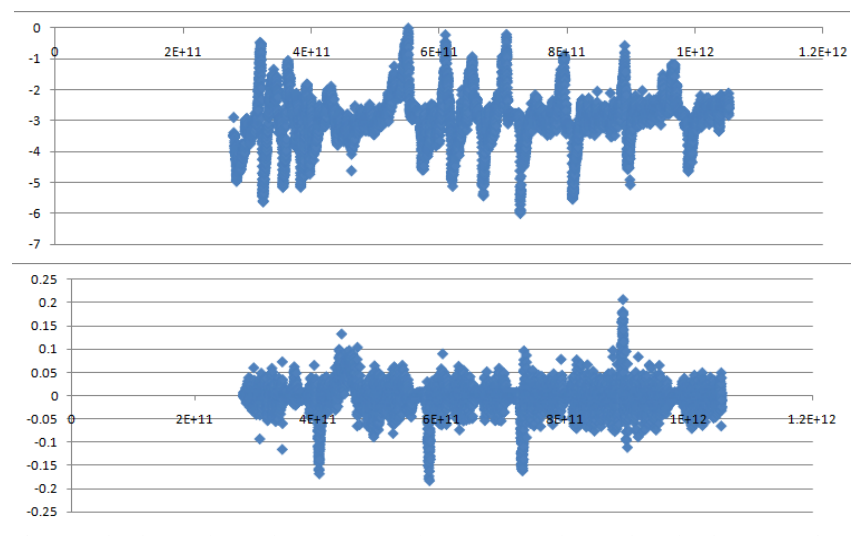

Fig. 2. The logged accelerometer and gyroscope data. They look very noisy.

- They gained substantial experiences on developing applications for the iOS and Android platforms.

- They experienced how to work in a collaborative research environment.

- They learned how to present research results, both in written and oral forms. Specifically, they prepared a poster and presented the posture in a min-conference organized by CSU for all sponsored undergraduate summer research projects.

Furthermore, students made very interesting observations in this project: (1) The GPS in both the Android and iOS devices is fairly accurate, as verified visually by superimposing the recorded trajectory with the map. (2) The method designed to improve the trajectory accuracy by fusing the GPS and inertial sensing data did not produce noticeable improvement. This is because the accelerometer and gyroscope data were too noisy to be useful, as shown in Fig. 2. (3) The sampling rate for GPS single is $1 \mathrm{~Hz}$, which also makes it difficult to fuse the GPS data with the accelerometer and gyroscope data, which have much higher sampling rate, due to the mismatch of sampling rates.

\section{AN AMERICAN Sign LANGUAGE TRANSLATION SySTEM BASED ON KINECT}

The American Sign Language (ASL) is being used by hundreds of thousands of hearing impaired persons in the US [9]. Unfortunately, it is virtually impossible for a hearing impaired person to communicate with a person without the disability (referred to as normal person from now on) without a human translator because very few normal persons have the training of ASL. This severely limits the social interactions of hearing impaired persons with the broader community. As such, it is very desirable to build low-cost compact systems that could translate between ASL and spoken English. This has been the research goal for numerous projects over the last decade (such as [10]).

Early generations of ASL to English translation systems were built primarily based on inertial sensors, which would require a hearing impaired person to wear custom-designed gloves equipped with sophisticated inertial sensors, or based on 2D cameras, which require the person to wear special colored gloves. Not only they are cumbersome to use, such systems are error-prone and supports very limited set of ASL words and expressions.

There was considerable excitement when the Kinect motion sensor [6] came on the market in 2010, offering a much less intrusive and more accurate means to capture the motion and expressions of a hearing impaired person. Indeed, several research groups and developers have been working toward an ASL-to-English translation system based on Kinect [10]. However, we have yet to see any significant breakthrough partially due to the limitations imposed by the early version of the Kinect sensor.

\section{A. Research Objectives}

ASL is a complex language that is expressed using hands movement, facial expression, hand shapes, body posture and head movement. Since the introduction of the Kinect sensor, both the hardware and software features have been added. The second version of the Kinect sensor (referred to as Kinect for Windows) is capable of performing facial recognition in addition to skeleton tracking, and a third party software development kit has been developed to recognize fingers [11], which makes the tracking of hand shapes possible. In the proposed project, we aim to address some of the limitations that the current research suffers:

- The use of a very small vocabulary of 10 words or less that involve only hands movement.

- Even with the small vocabulary, only individual signs are recognized instead of complete sentences that consist of a number of signs.

- Research on recognizing hand-shapes is limited to static signs only.

- There is no consideration of grammatical features of ASL expressed using body posture, head movement, and facial expressions.

In particular, we plan to explore the translation of whole sentences of ASL by tracking all elements that are needed to understand them, including the movement of hands, the shapes of the hand, body posture, head movement, and facial expressions. In addition, we plan to develop an application that can do two-way translations, i.e., not only the sign language can be recognized and translated into English, spoken or written English can also be translated visually into ASL via a computer or TV monitor.

\section{B. Specific Research Duties of Undergraduate Students}

According to our plan, two undergraduate students will engage carry out three groups of tasks. The first group of tasks focuses on the sign language recognition, and they were assigned to one of the two students. The second group of tasks focus on developing software to render sign language visual display based on spoken English, which were assigned to the other student. The third group of tasks is common tasks assigned to both students.

Group one tasks:

1) Getting familiar with the facial recognition application programming interfaces (APIs).

2) Getting familiar with the hands tracking [11] APIs (offered by a $3^{\text {rd }}$ party package called SigmaNIL [12]).

3) Getting familiar with the skeleton tracking APIs. 
4) Able to perform single signage recognition

5) Whole sentence recognition with consideration of facial expressions and body postures, etc.

Group two tasks:

1) Getting familiar with the voice recognition APIs.

2) Getting familiar with video rendering and bitmap manipulation APIs.

3) Investigating the best way to render the sign language motions visually translated from English. We will explore two possibilities: (1) build a database for common sign language phrases/sentences, and (2) rely on on-the-fly animations based on pre-accumulated hand positions, skeleton, and facial expression data.

Group three tasks:

1) Studying ASL to acquire basic understanding of ASL.

2) Recording videos and capturing data to build a database for English-ASL two-way translations.

\section{Outcomes}

The students were able to learn some basic American Sign Language with a help of another student majoring in special education (task 9). They were also able to develop a Kinect application for recording ASL demonstration and built a dataset of several dozen ASL phrases (task 10, task 7).

They were also able to develop a separate Kinect application for demonstrating the translation between ASL and English, as shown in Fig. 3. They experimented with using a machine-learning based approach for ASL recognition. Unfortunately, the effort was not successful and the recognition rate is rather low. This could be attributed to the fact that the students were not familiar with the proper use of machine-learning algorithms, and did not know how to select feature vector for the recognition. In our curriculum, no machine learning related course is offered. As the result, they failed to complete the remaining tasks.

Nevertheless, the students did learn various APIs and were able to develop a Kinect application, and learn how to collect and analyze Kinect skeleton data.

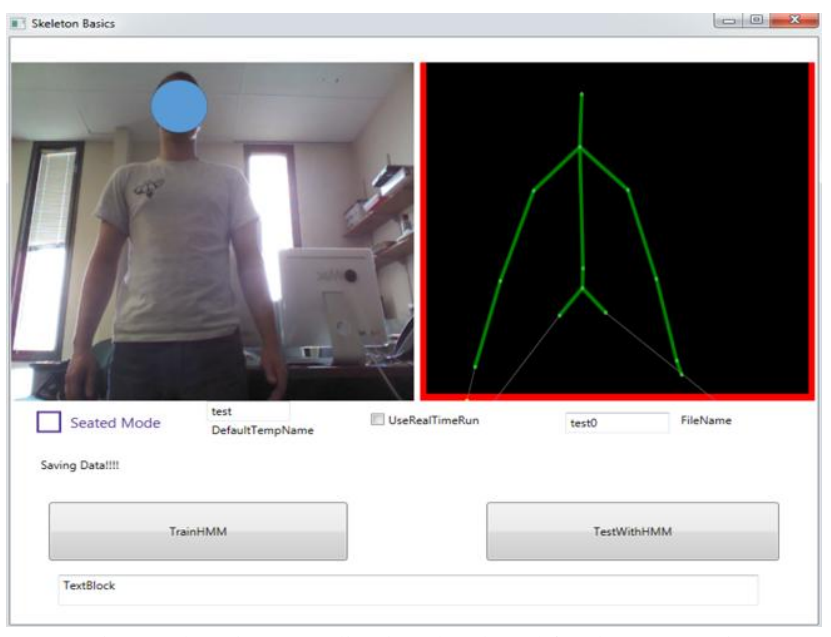

Fig. 3. The Kinect application developed for the ASL project.

\section{A Low COST Motion ANALYSIS System BASED ON KINECT}

Up to a decade ago, it was not uncommon for motion analysis to be used clinically in the assessment of individuals with complex neurologic dysfunction or orthopedic conditions. This now occurs infrequently. In clinical medicine, a motion analysis study costs in the range of $\$ 1,500$ and is rarely covered by health insurance. The systems are not highly accessible to patients since they are typically located in major medical centers or universities due to their high cost $(>\$ 80,000)$. In addition, they are not user friendly, requiring significant amounts of training time in order to learn operation and analysis skills. While real time systems have considerably decreased analysis time, this time commitment remains substantial. Therefore, there was considerable excitement when the Kinect motion sensor [13] came on the market in 2010, offering an inexpensive and therefore more accessible motion capture system that requires minimal training to use. Investigation has already begun in using the Kinect system for balance training post neurologic injury [14], gait analysis, home-based falls assessment, hand motion analysis, and chronic pain rehabilitation [15].

\section{A. Research Objectives}

We hypothesize that the Kinect system will provide accurate kinematic data for large excursion movements but may require the addition of inertial sensors for smaller amplitude motions, and for motions that involve the blockage of some body joints. We plan to test the accuracy of the Kinect motion sensors and establish when it is necessary resort to the inertial sensor system to obtain accurate motion data. This summer project is the first step in the integration of these two sensor technologies with the ultimate goal of providing a low cost motion analysis technology. Specifically, this summer we propose to examine the accuracy and the limitations of the joint kinematic data collected by the Kinect system. We will do this analysis by comparing Kinect motion data with the gold standard eight-camera three-dimensional motion analysis data using the CSU School of Health Science's Motion Analysis Lab.

One difficulty with the analysis of human motion data is that human movement is extremely variable and complex. In order to test the ability of the sensor system to accurately monitor movement, a representative group of motions must be selected to represent this wide range of possible motions. For the purposes of this initial proposal, we will examine only gross motor activities and activities that occur "in place", such as a movement done in sitting or standing. The motions we will examine vary along the continua of body position, velocity of movement, acceleration, amplitude/excursion of movement, rotational motion, and segmental alignment. Six different activities have been selected to represent various critical aspects of these movement continua, namely hamstring stretch, can turn, sit to stand, lateral weight shift, video game bowling, and hip abduction. These six activities will be tested with concurrent collection of kinematic motion data from the Kinect and eight-camera motion analysis systems. Data will be analyzed from each system and compared statistically.

\section{B. Specific Research Duties of Undergraduate Students}

According to our plan, three undergraduate students will engage in various aspects of developing a low-cost motion analysis system based on Kinect. More specifically, the 
students will carry out the following tasks:

- Help prepare the Institutional Review Board (IRB) proposal and human subject recruitment, as well as learning how to collect and analyze kinematic Motion Lab data.

- Learn how to collect data with the Kinect system and write the code necessary for analysis of this data.

- Help identify the limitations of the Kinect sensor.

- Assist with the preparatory piloting of data collection and analysis, followed by the actual data collection and statistical analysis.

\section{Outcomes}

The students were able to accomplish virtually all research tasks. The students were able to adapt an existing Kinect application provided by Microsoft for the purpose of data logging. They also learned the process of working with human subjects, including IRB proposal and subject recruitment, and how to collect and perform preliminary analyze the data.

Specifically, the following observations were made: (1) The Kinect sensor reports very accurate positions for moving joints and relatively accurate positions for stationary joints, as shown in Fig. 4.

Furthermore, the use of aggressive smoothing technique can improve the accuracy of the stationary joints positions, as shown in Fig. 5.

However, for motions involving subtle rotations, it is beyond the capability of the Kinect. This limitation is important because to characterize the quality of the exercise, it is essential to identify places where transverse plane rotation is needed or should be avoided.
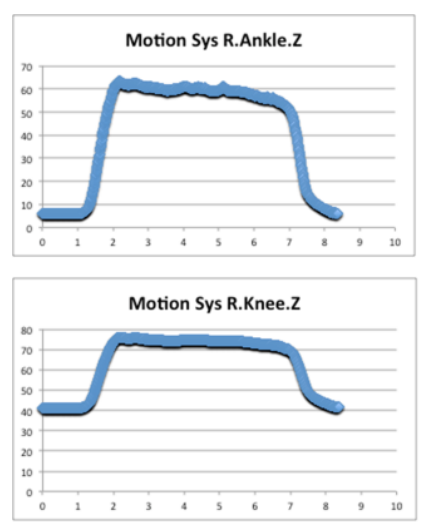

Fig. 4. Comparison of the joint measurement by Kinect (right side) and the multi-camera motion analysis system (left side).

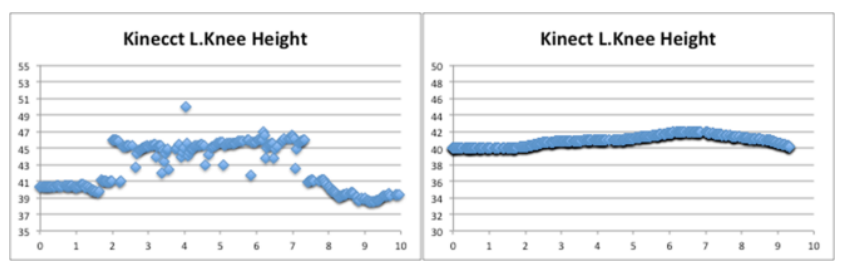

Fig. 5. Raw Kinect joint data (left) and the corresponding smoothed data.

\section{TOWARDS SAFER PATIENT HANDLING AMONG WORKERS IN NURSING HOMES}

Several studies have shown that workers at nursing homes (i.e., certified nursing assistants, or STNA's) have a higher risk of lower back musculoskeletal injuries because they have the primary responsibility for heavy lifting in patient handling. Such injuries often result in significant lost work time, and could lead to low morale in workplace. While best practices in patient handling are known, training alone has proven ineffective due to the lack of compliance in actual practice [16], [17]. In this project, we aim to develop a low-cost real-time monitoring and feedback system to enhance the workers' compliance to best practices. The system will relies on the integration of two cutting edge technologies:

1) The Microsoft Kinect technology, which enables computer-vision based human motion tracking and activity recognition [18]; and

2) The Smart Watch technology, which enables haptic feedback to the worker who wears such a smart watch [19].

\section{A. Research Objectives}

While many Kinect applications have been developed in the past, and similarly many smart watch applications have been developed, we are not aware of any research work on integrating the two technologies towards a motion tracking system with real-time haptic feedback. The integration of the two technologies also addresses the privacy concerns of the patients: only the worker who is wearing the smart watch will be tracked. Specifically, this project consists of the following research objectives:

- Create a system that monitors, identifies, and tracks appropriate use of patient over-bed lift systems and related worker body mechanics. Specifically, we want to (1) identify appropriate vs. lack of use of over bed lifts for patient lifting; (2) determine and implement hardware needs for sensors to capture necessary data; and (3) identify correct vs. incorrect body movements during patient handling in real-time.

- Create a system for alerts and overall feedback that prompts workers in real-time and provides knowledge of their performance over time about their use of lifts and correct body mechanics. Specifically, we would like to (1) identify needs for worker education as to the use of lifts and body mechanics during patient handling, including perceived barriers to and promoters of their use; (2) to identify the most acceptable (to the workers) and effective parameters for the real-time and cumulative sensor feedback and alert system to encourage adherence to best practices for patient handling; and (3) to identify the perceptions towards and acceptance of the workers to the real-time and cumulative (across time) feedback system, including barriers to and promoters of its use.

In the addition to the above high-level tasks, we aim to address the following specific research challenges:

- Synchronization of the smart watch and the Kinect motion tracking via gesture recognition in both components. This requires us to investigate methods to compare motions captured by two completely different tracking technologies, one based on direct skeletal tracking (Kinect), and the other based on inertial sensors (smart watch).

- Two-way communications between a smart watch and the Kinect application running in a computer system. Many smart watches are only equipped with Bluetooth 
and are incapable of directly communicating with computer system, in which the Kinect application must reside. Hence, the two-way communication between the smart watch and the Kinect application must be mediated via a mobile app running in a smart phone.

\section{B. Specific Research Duties of Undergraduate Students}

Two undergraduate students are expected to engage in various aspects of developing the real-time motion monitoring and feedback system. More specifically, the students will carry out the following tasks:

- One student will be in charge of reviewing established safe patient handling protocols and compile a list of ergonomic peripherals used with overhead lift system, which our lab is equipped with and is used in our external partner site. The student will also help define the safe patient handing rules, as well as learn how to collect and analyze data obtained by the proposed system.

- Another student will be in charge of carrying out the computer programming tasks listed in the previous section for the development of the proposed system. Specifically, the student will engage in the programming of three types of platforms: 1) Kinect (for motion tracking); 2) Pebble smart watch (for motion tracking, syncing, and feedback); and 3) a mobile app (for mediation of Kinect application and the watch app).

\section{Outcomes}

The students in the project were able to complete all planned tasks. The primary research outcome is a low-cost motion tracking and real-time haptic feedback system. The system architecture and the demonstration interface are shown in Fig. 6 and Fig. 7, respectively.
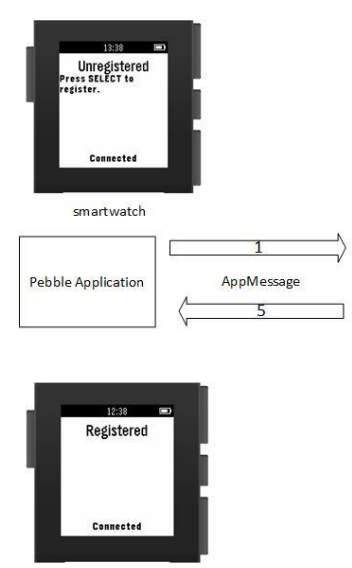

Fig. 6. The privacy-aware human motion tracking system.

In addition to the research outcomes, the undergraduate student participants accomplished the following:

- Learn the patient safe handling best practices and polices

- Learn the existing ergonomic equipment available towards safer patient handling in nursing homes

- Learn how to program Microsoft Kinect sensors

- Learn how to program Pebble smart watches

- Learn how to perform action recognition using both Kinect and inertial sensors in the smart watch, and as well as data logging

- Learn how to develop a real-time communication service for haptic and text-based feedback to a worker for safer patient handling

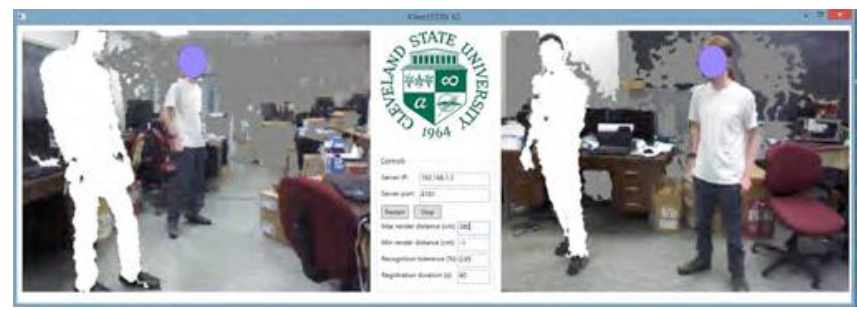

Fig. 7. The demonstration interface for the privacy-aware human motion tracking system.

In addition to completing the proposed research tasks, the students in the project made very impressive unexpected research contributions. For example, after implementing the proposed registration mechanism for selective computer vision tracking, which relies in part on the detection of the tapping gesture using inertial sensors equipped in the Pebble smart watch, the students observed that the detection mechanism on the smart watch did not work well. They subsequently came up with a much more reliable approach to register after consulting with us. Instead of detecting the gesture using the inertial sensors, they altered the gesture slightly from tapping to bushing a button. The detection of pushing of a button is directly detected via the built-in hardware. Hence, it is much more reliable.

Furthermore, the team received an invitation from One Community, a local non-profit organization, to demonstrate our project in the [R] IoT Happy Hour event in July 2015. The demonstration attracted many visitors and was very successful.

This project was highly successful. The research results have led to one paper to be presented at an IEEE conference [20], and a more comprehensive article submitted to an IEEE Transactions. Both undergraduate students are co-authors of the papers.

\section{CONCLUSION}

In this paper, we shared our effort in enhancing undergraduate research experiences for students at the Cleveland State University. We showcased four summer research projects sponsored by the CSU Office of Research. Due to lack of experience, the early projects (the first two projects described in this paper) were not completely successful in that students could only partially complete the projects. As we gained experience, recent projects (the last two projects described in this paper) were properly designed for undergraduate students, which have led to excellent results. These last two projects have directly and indirectly resulted in numerous peer-reviewed publications [20]-[25], some of which were co-authored with undergraduate students.

\section{ACKNOWLEDGMENT}

We thank the anonymous reviewers for their invaluable comments on an earlier version of this paper. We would like to thank the following students for participating and contributing to the projects described in this article: Arnold Csok, Steven Reba, Sam Yokoyama, Vitaliy Sinyuk, Kelsey 
Bujdos, Tracy Jennemann, Vitaliy Sinyuk, Sam Yokoyama, Connor Gordon and Abou-Bakar M. Fofana.

\section{REFERENCES}

[1] D. Lopatto, "Survey of undergraduate research experiences (SURE): First findings," Cell Biology Education, vol. 3, no. 4, pp. 270-277, 2002.

[2] D. Lopatto, "Undergraduate research experiences support science career decisions and active learning," CBE-Life Sciences Education, vol. 6, no. 4, pp. 297-306, 2007.

[3] A. L. Zydney, J. S. Bennett, A. Shahid, and K. W. Bauer, "Impact of undergraduate research experience in engineering," Journal of Engineering Education, vol. 91, no. 2, pp. 151-157, 2002

[4] A. L. Zydney, J. S. Bennett, A. Shahid, and K. W. Bauer, "Faculty perspectives regarding the undergraduate research experience in science and engineering," Journal of Engineering Education, vol. 91, no. 3, pp. 291-297, 2002.

[5] L. Reisberg, "Research by undergraduates proliferates, but is some of it just glorified homework," The Chronicles of Higher Education, vol. 20 pp. A45-A46, 1998.

[6] P. Mohan, V. Padmanabhan, and R. Ramjee, "Nericell: Rich monitoring of road and traffic conditions using mobile smartphones," in Proc. SenSys '08, Raleigh, NC, November 5-7, 2008.

[7] C. Ziegler. (February 2011). Boston planning to murder potholes with your phone's accelerometer. Engadget. [Online]. Available: http://www.engadget.com/2011/02/13/boston-planning-to-murder-pot holes-with-your-phones-acceleromet/

[8] A. C. Harvey, Forecasting, Structural Time Series Models and the Kalman Filter, Cambridge University Press, 1990.

[9] W. C. Stokoe, "Sign language structure: An outline of the visual communication systems of the American deaf," Journal of Deaf Studies and Deaf Education, vol. 10, no. 1, pp. 3-37, 2005.

[10] S. Lang, M. Block, and R. Rojas, "Sign language recognition using Kinect," LNCS, vol. 7267, 2012, pp. 394-402.

[11] J. Raheja, A. Chaudhary, and K. Singal, "Tracking of fingertips and centers of palm using kinect," in Proc. the Computational Intelligence, Modelling and Simulation, pp. 248-252, 2011.

[12] Sigmanil framework. OpenNI. [Online]. Available: http://www.openni.org/files/sigmanil-framework/

[13] Microsoft. Meet Kinect for Windows. [Online]. Available: https://developer.microsoft.com/en-us/windows/kinect

[14] B. Lange, C. Y. Chang, E. Suma, B. Newman, A. S. Rizzo, and M. Bolas, "Development and evaluation of low cost game-based balance rehabilitation tool using the Microsoft Kinect sensor," in Proc. the Annual International Conference of the IEEE Engineering in Medicine and Biology Society, EMBC, pp. 1831-1834. IEEE, 2011.

[15] C. Schonauer, T. Pintaric, H. Kaufmann, S. Jansen-Kosterink, and M. Vollenbroek-Hutten, "Chronic pain rehabilitation with a serious game using multimodal input," in Proc. the International Conference on Virtual Rehabilitation, pp. 1-8, 2011.

[16] A. Garg and J. M. Kapellusch, "Long-term efficacy of an ergonomics program that includes patient-handling devices on reducing musculoskeletal injuries to nursing personnel," Human Factors: The Journal of the Human Factors and Ergonomics Society, vol. 54, no. 4, pp. 608-625, 2012.

[17] B. D. Owen, K. Keene, and S. Olson, "An ergonomic approach to reducing back/shoulder stress in hospital nursing personnel: a five year follow up," International Journal of Nursing Studies, vol. 39, no. 3, pp 295-302, 2002

[18] R. Lun and W. Zhao, "A survey of applications and human motion recognition with Microsoft Kinect," International Journal of Pattern Recognition and Artificial Intelligence, vol. 29 no. 05, pp. 1555008, 2015.

[19] W. Zhao, D. D. Espy, M. Reinthal, B. Ekelman, G. Goodman, and J. Niederriter, "Privacy-aware human motion tracking with realtime haptic feedback," in Proc. the IEEE International Conference on Mobile Services, pp. 446-453, 2015.

[20] W. Zhao, R. Lun, C. Gordon, A.-B. Fofana, D. D. Espy, M. Reinthal, B. Ekelman, G. Goodman, J. Niederriter, C. Luo, and X. Luo, "A privacy-aware Kinect-based system for healthcare professional," in Proc. the IEEE International Conference on Electro/ Information Technology, Grand Forks, North Dakota, USA, May 19-21, 2016.

[21] W. Zhao, R. Lun, D. D. Espy, and M. A. Reinthal, "Realtime motion assessment for rehabilitation exercises: Integration of kinematic modeling with fuzzy inference," Journal of Artificial Intelligence and Soft Computing Research, vol. 4, no. 4, pp. 267-285, 2015.

[22] W. Zhao, D. Espy, M. A. Reinthal, and H. Feng, "A feasibility study of using a single kinect sensor for rehabilitation exercises monitoring: A rule based approach," in Proc. the IEEE Symposium on Computational Intelligence in Healthcare and e-Health, Orlando, Florida, USA, December 9-12, 2014, pp. 1-8.

[23] W. Zhao, R. Lun, D. Espy, and M. A. Reinthal, "Rule based realtime motion assessment for rehabilitation exercises," in Proc. the IEEE Symposium on Computational Intelligence in Healthcare and e-Health, Orlando, Florida, USA, December 9-12, 2014, pp. 133-140.

[24] W. Zhao, H. Feng, R. Lun, D. Espy, and A. Reinthal, "A kinect-based rehabilitation exercise monitoring and guidance system," in Proc. the 5th IEEE International Conference on Software Engineering and Service Science, Beijing, China, June 27-29, 2014, pp. 762-765.

[25] W. Zhao, D. Espy, A. Reinthal, and H. Feng, "Feasibility study of using microsoft kinect for physical therapy monitoring," Encyclopedia of Information Science and Technology, 3rd ed. 2014, pp. 5542-5554.

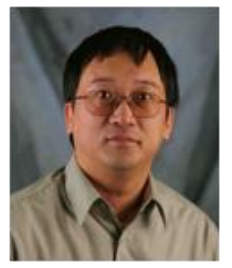

Wenbing Zhao received his Ph.D. in electrical and computer engineering at University of California, Santa Barbara, under the supervision of Drs. Moser and Melliar-Smith, in 2002. Dr. Zhao has a bachelor of science degree in physics in 1990, and a master of science degree in physics in 1993, both at Peking University, Beijing, China. Dr. Zhao also received a master of science degree in electrical and computer engineering in 1998 at University of California, Santa Barbara.

From 2000 to 2004, he worked as a senior software engineering and chief architecture at Eternal Systems, Inc. From 2003-2004, he held a concurren position as a post-doctoral researcher at the University of California, Santa Barbara. From 2004 to the present, he has worked first as an assistant professor, and then as a tenured associate professor. He has recently been promoted to the full professor rank effective fall 2016. He is currently serving as the director of the master of science in electrical engineering, and the chair of the graduate program committee in the Department of Electrical Engineering and Computer Science.

Dr. Zhao has authored a research monograph titled: "Building Dependable Distributed Systems" published by Scrivener Publishing, an imprint of John Wiley and Sons. Furthermore, Dr. Zhao published over 120 peer-reviewed papers in the area of fault tolerant and dependable systems (three of them won the best paper award), computer vision and motion analysis, and material sciences. Dr. Zhao's research is supported in part by the US National Science Foundation, the US Department of Transportation, Ohio State Bureau of Workers' Compensation, and by Cleveland State University. Dr. Zhao is currently serving on the technical program committee for numerous international conferences and is a member of editorial board for PeerJ Computer Science, International Journal of Parallel Emergent and Distributed Systems, International Journal of Distributed Systems and Technologies, International Journal of Performability Engineering, International Journal of Web Science, and several international journals of the International Academy, Research, and Industry Association. Dr. Zhao is a senior member of IEEE and is currently serving on the executive board of the IEEE Cleveland Section. Dr. Zhao is also a senior member of International Economics Development and Research Center (IEDRC). 